\title{
Lack of spatial structure for phenotypic and genetic variation despite high self-fertilization in Aquilegia canadensis (Ranunculaceae)
}

\author{
MP Bartkowska $^{1} \cdot$ AY-C Wong $^{1} \cdot$ SP Sagar $^{1} \cdot$ L Zeng $^{1} \cdot$ CG Eckert $^{1}$
}

Received: 11 October 2017 / Revised: 13 January 2018 / Accepted: 18 January 2018 / Published online: 1 March 2018

(c) The Genetics Society 2018

\begin{abstract}
By reducing genetically effective population size and gene flow, self-fertilization should lead to strong spatial genetic structure (SGS). Although the short-lived plant Aquilegia canadensis produces large, complex, nectar-rich flowers, $75 \%$ of seed, on average, are self-fertilized. Previous experimental results are consistent with the fine-scale SGS expected in selfing populations. In contrast, key floral traits show no evidence of SGS, despite a significant genetic basis to phenotypic variation within populations. In this study, we attempt to resolve these contradictory results by hierarchically sampling plants from two plots nested within each of seven rock outcrops distributed over several $\mathrm{km}$, and comparing the spatial pattern of phenotypic variation in four floral traits with neutral genetic variation at 10 microsatellite loci. For both floral and microsatellite variation, we detected only weak hierarchical structuring and no isolation by distance. The spatial pattern of variation in floral traits was on par with microsatellite polymorphisms. These results suggest regular long-distance gene flow via pollen. At much finer spatial scales within plots, estimates of relatedness were higher (albeit very low) between nearest neighbors than random plants, and declined with increasing distance between neighbors, which is consistent with highly localized seed dispersal. High selfing should yield SGS, but strong inbreeding depression in A. canadensis likely erodes SGS so that reproductive plants exhibit weak structure typical of outcrossers, especially given that outcrossing and consequent gene flow in this species are mediated by strong-flying hummingbirds and bumble bees.
\end{abstract}

\section{Introduction}

The spatial distribution of genetic variation within a species is expected to be shaped by antagonism between processes increasing genetic differentiation and those promoting genetic homogeneity (Sexton et al. 2013). For neutral genetic variation, this amounts to genetic drift increasing differentiation, whereas gene flow generally reduces it. Across the geographic distribution of populations, this often leads to the classic pattern of isolation by distance (IBD), where populations that are in closer spatial proximity are

Electronic supplementary material The online version of this article (https://doi.org/10.1038/s41437-018-0065-2) contains supplementary material, which is available to authorized users.

CG Eckert

chris.eckert@queensu.ca

1 Department of Biology, Queen's University, Kingston, ON K7L 3N6, Canada also more genetically similar. The balance between drift and gene flow will, in turn, be influenced by aspects of life history, reproductive system and dispersal that affect the movement of individuals (or their gametes) and the distribution and demography of populations (Loveless and Hamrick 1984; Hamrick and Godt 1996; Glémin et al. 2006). For genetic variation that influences fitness, however, the pattern of spatial genetic structure (SGS) may also be influenced by selection.

The mode and direction of selection may differ in space and time with a variety of ecological factors that influence the relation between genetically-based trait variation and fitness (reviewed in Sexton et al. 2013). Spatial genetic structure may be reduced if similar selection pressures act across populations. Alternatively, local variation in selective pressures may increase SGS as a result of local adaptation. Insight into the role of selection in determining phenotypic SGS can be had by comparing the SGS of traits, or ecologically significant alleles, with that of selectively neutral genetic polymorphisms (Brommer 2011 and references therein). 
Diverse manifestations of this general approach have shown that selection can also shape SGS by influencing phenotypic divergence among populations. For example, Schemske and Bierzychudek (2007) observed striking spatial variation in the frequency of genetically determined flower color morphs in Linanthus parryii that contrasted with spatial homogeneity in putatively neutral allozyme polymorphisms, implying selection causing SGS in flower color. More recently, studies have formalized this approach by comparing standardized measures of differentiation in phenotype $\left(P_{\mathrm{ST}}\right)$ with population differentiation for putatively neutral polymorphisms $\left(F_{\mathrm{ST}}\right.$, e.g., DeFaveri and Merilä 2013; Brommer 2011), with the latter providing the null expectation of differentiation under drift and gene flow alone. The inferential power of this approach can be increased by using spatially hierarchical sampling designs because the processes acting on genetic variation may vary with spatial scale (Schoville et al. 2012).

Spatial genetic structure and the extent to which it can be interpreted in terms of selective vs. neutral processes will be strongly influenced by the mating system and dispersal biology (reviewed in Hamrick and Godt 1996; Wright et al. 2013). Most plants are hermaphroditic and thus many engage in substantial self-fertilization. Increased homozygosity within selfing populations reduces effective population size, thereby enhancing genetic drift (Wright et al. 2013). Selfing also reduces opportunities for gene flow via pollen (Ingvarsson 2002), which is generally thought to be the most important mode of long-distance gene movement in plants (Petit et al. 2005; Ellstrand 2014). Enhanced genetic drift coupled with reduced gene flow will generate genome-wide SGS.

The effect of selfing on population genetic structure, especially for later stages in the life cycle, will be modified by the relative fitness of selfed compared to outcrossed offspring (inbreeding depression). Strong inbreeding depression will tend to make the SGS of a partially selfing population resemble that of a more outcrossing population because offspring produced by outcrossing become more frequent among surviving and reproducing individuals (Hufford and Hamrick 2003, see also Brunet et al. 2012). Hufford and Hamrick (2003) documented the genetic composition of fertilized ovules, seeds, and seedlings within a cohort and showed an overall decrease in self-fertilization at later life history stages, such that the adult population did not differ from Hardy-Weinberg Equilibrium. Studies showing shifts in relatedness from juvenile to later life history stages also provide inferential support of the hypothesis that inbreeding depression can alter the genetic structure within a population (Cruse-Sanders and Hamrick 2004). Studies that explicitly contrast SGS for different traits/loci in species where the degree of self-fertilization and the strength of inbreeding depression are known are needed to establish under what conditions inbreeding depression can alter SGS and at what scale the effects manifest (but see Hufford and Hamrick 2003; CruseSanders and Hamrick; Brunet et al. 2012).

In this study, we contrast the spatial pattern for phenotypic variation in floral traits with that for neutral microsatellite polymorphisms in the short-lived, herbaceous plant Aquilegia canadensis L. (Ranunculaceae). Although the species invests heavily in large, complex, nectar-rich flowers that appear adapted for pollination by hummingbirds (Macior 1996) - pollinators capable of mediating long-distance gene flow-genetic analysis of progeny arrays from populations throughout the geographic range (including many populations from the region studied here) indicate that most populations are mixed-mating and predominantly self-fertilizing (on average the proportion of selfed seeds is 0.75 and ranges $0.15-1.00, n=63$ population-year estimates, Eckert and Herlihy 2004). Moreover, this species typically occurs in small, isolated aggregations on patchily distributed rock outcrops and lacks any obvious traits for seed dispersal.

Together, small patchy populations, limited dispersal and frequent self-fertilization would promote the development of SGS in A. canadensis. However, inbreeding depression appears to be very strong in this species, as allozyme-based inbreeding coefficients $(F)$ of reproductive plants are very low despite most seeds being produced through self-fertilization, suggesting that selfed offspring rarely survive to maturity (Eckert and Herlihy 2004). Strong inbreeding depression can dramatically increase the contribution of outcrossing to population genetic structure thereby increasing $N_{\mathrm{e}}$ and gene flow (Yang and Hodges 2010), especially if outcrossing events are mediated by large, strong-flying pollinators like hummingbirds (Macior 1996).

Previous studies have produced conflicting evidence regarding the extent of SGS in natural populations of $A$. canadensis. Evidence from two different approaches is consistent with fine-scale genetic structure, suggesting that relatives tend to be closely clustered and gene flow is limited. First, flowers rendered phenotypically female through experimental emasculation exhibited substantial biparental inbreeding consistent with fine-scale genetic structure and localized pollen movement (Herlihy and Eckert 2004). Second, Griffin and Eckert (2003) showed that moving plants to random locations within populations, which would break up fine SGS, significantly reduced inbreeding (Griffin and Eckert 2003). In contrast, phenotypic variation in key floral traits, of which a significant component has a genetic basis (Herlihy and Eckert 2007), does not exhibit spatial structuring at local scales $(\sim 10-50 \mathrm{~km})$. For three populations in the study area used here, broad-sense heritability 
$\left(H^{2}\right)$ estimates were moderate to high (herkogamy: 0.21-0.78, pistil length: $0.04-0.31$ and spur length: 0.16-0.32). Variation in key floral traits was high among individuals within populations but low between populations (Herlihy and Eckert 2007). Differentiation in floral morphology among populations of A. canadensis was only evident across very large spatial scales (1000's of km; Herlihy and Eckert 2005). Although the lack of spatial structure at smaller spatial scales could be attributed to uniform stabilizing selection, this is inconsistent with the large amount of heritable floral variation within A. canadensis populations (Herlihy and Eckert 2007). This suggests that gene flow is much more extensive than suggested by the experimental studies discussed above.

Here we reconcile the conflicting observations of Herlihy and Eckert (2004) and Griffin and Eckert (2003) with those of Herlihy and Eckert (2007) by directly comparing hierarchical SGS in both putatively neutral microsatellite polymorphisms vs. genetically-based phenotypic variation in floral traits. We sampled individuals hierarchically so that variation for floral traits and neutral genetic markers can be estimated among plants separated by meters-where evidence of SGS was documented by Griffin and Eckert (2003) and Herlihy and Eckert (2004) — among plots separated by tens of meters within local populations and among populations separated by hundreds of meters to kilometers. We compare the statistical partitioning of variance at these hierarchical levels between heritable morphological trait variation and marker genes. Similar partitioning would imply similar evolutionary processes acting on SGS. We also test whether the two types of variation exhibit similar patterns of increasing differentiation with increasing geographic distance (isolation by distance) as would be expected from the opposing effect of genetic drift vs. gene flow.

\section{Materials and methods}

\section{Study organism and sampling design}

Aquilegia canadensis produces large, red, nodding flowers consisting of 4-6 petals modified into narrow, long $(\sim 30 \mathrm{~mm})$ nectar spurs and the same number of long $(\sim 20 \mathrm{~mm})$ sepals. Each flower contains a gynoecium of $\sim 5$ fused carpels each containing $\sim 20$ ovules, which is surrounded by a column of $30-40$ stamens that elongate asynchronously throughout floral anthesis. Stigmas are usually exserted beyond the dehiscing anthers and become receptive when the first anthers dehisce. In southeastern Ontario, where this study was conducted (Supplementary Figure S1), flowering occurs mid-to-late spring (May-June) and seeds mature in early summer (mid July). Ruby-
Table 1 Hierarchical sampling for floral morphology and allele frequencies at nine microsatellite loci of Aquilegia canadensis in two plots from each of seven outcrops

\begin{tabular}{llllll}
\hline Outcrop & Plot & $\begin{array}{l}\text { Flowers } \\
\text { measured }\end{array}$ & $\begin{array}{l}\text { Plants } \\
\text { genotyped }\end{array}$ & $\begin{array}{l}\text { Grid } \\
\text { position E } \\
(\mathrm{m})\end{array}$ & $\begin{array}{l}\text { Grid } \\
\text { position N } \\
(\mathrm{m})\end{array}$ \\
\hline QBED1 & 1 & 23 & 20 & 390901 & 4934429 \\
& 2 & 23 & 20 & & \\
QBED2 & 1 & 25 & 20 & 390597 & 4933875 \\
& 2 & 22 & 20 & & \\
QLL1 & 1 & 24 & 20 & 390534 & 4932248 \\
& 2 & 23 & 20 & & \\
QLL3 & 1 & 25 & 20 & 389812 & 4932584 \\
& 2 & 27 & 20 & & \\
QRL1 & 1 & 22 & 20 & 390566 & 4930677 \\
& 2 & 28 & 20 & & \\
QRL2 & 1 & 27 & 20 & 390573 & 4930642 \\
& 2 & 21 & 20 & & \\
QTUR & 1 & 26 & 20 & 390646 & 4933399 \\
& 2 & 25 & 20 & & \\
\hline
\end{tabular}

Outcrops were open granite domes of area $100-1000 \mathrm{~m}^{2}$, with 479-3801 m between outcrops, and $\sim 30 \mathrm{~m}$ between $20 \mathrm{~m}$ diameter plots within outcrops

The Universal Transverse Mercator (UTM) coordinates are in meters. All outcrops were inside UTM zone 18T.

throated hummingbirds (Archilochus colubris) and several species of long-tongued bumble bees (Bombus spp.) are the main flower visitors in this part of the species range. Pollinator visitation to individual flowers is, however, very infrequent.

We sampled A. canadensis from seven rock outcrops during spring 2008 (Table 1). Outcrops were open granite domes of area $100-1000 \mathrm{~m}^{2}$, and distances between outcrops ranged $479-3801 \mathrm{~m}$. For two outcrops used in this study (QLL1 and QLL3), previous estimates of the proportion of seeds self-fertilized based on progeny arrays (0.65-0.80 for QLL1 and 0.60-0.65 for QLL3, Eckert and Herlihy 2004) indicate that like elsewhere in the species geographic range, populations tend to be mixed mating and predominantly selfing. On each outcrop, we nested two 20$\mathrm{m}$ diameter circular plots located $\sim 30 \mathrm{~m}$ apart (center to center). Within each plot, we randomly selected 21-28 plants for which we measured four floral traits on one randomly selected flower and collected a basal leaf that was flash dried in silica for genetic analysis. We genotyped $\sim 20$ plants per plot ( $n=280$ plants total, Table 1, Fig. 1).

\section{Floral measurements}

For each flower, we measured (to $0.1 \mathrm{~mm}$ ) the length of one nectar spur, from the distal edge of the nectar gland to the 
Fig. 1 Population structure inferred by InStruct Bayesian clustering of Aquileqia canadensis assayed for nine microsatellite loci. Population assignments for 280 individuals at cluster number $K=2$ (left plot) with sampling plots ordered from north (top) to south (bottom), and geographic location of the sampled outcrops (right plot). The geographic location of the study area is indicated in Supplementary Figure S1
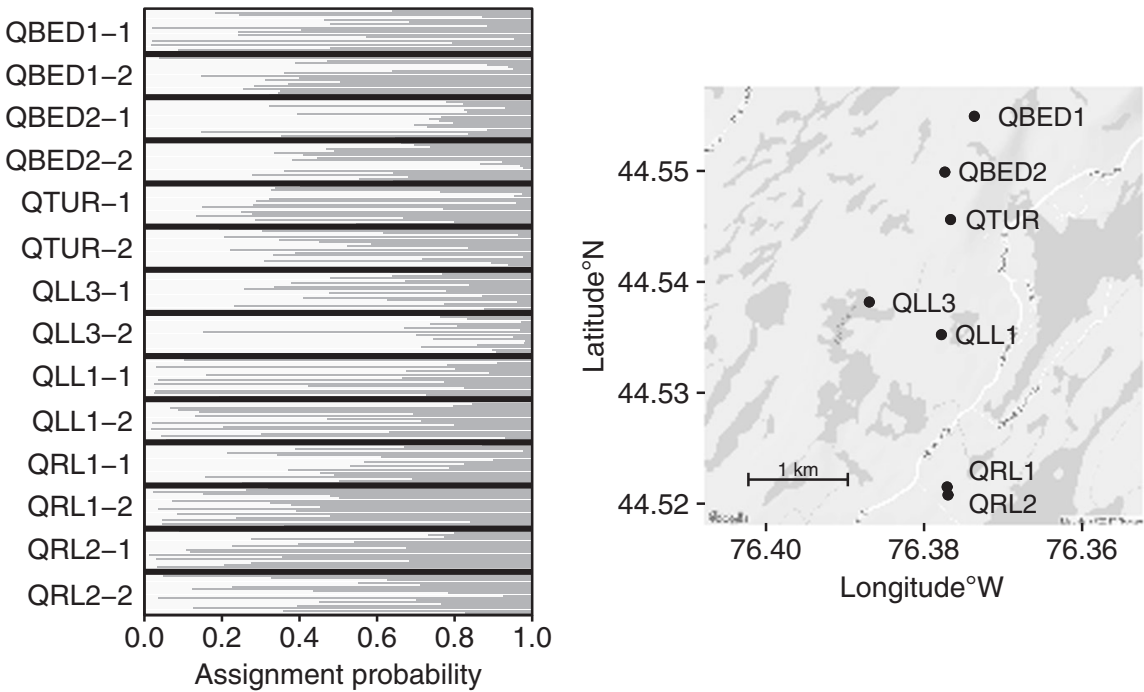

tip of the lamella, the length of one sepal, from tip to the junction with the floral column, the length of one pistil from receptacle to stigma, and herkogamy as the minimum distance between a receptive stigma and a dehiscing anther. Because styles elongate during the floral lifespan, thereby increasing herkogamy (Griffin et al. 2000), we tried to measure flowers at a standard developmental stage (after dehiscence of the $10^{\text {th }}$ anther). We recorded how many anthers were dehisced as a measure of floral age and noted whether the flower was borne on a primary, secondary or tertiary shoot as some floral traits vary with rank on the inflorescence (Kliber and Eckert 2004). To estimate measurement error, each trait was measured twice in a random order. These measurements were made by three observers, whose sampling effort was haphazardly distributed among plots and outcrops.

\section{Genetic assays}

We extracted genomic DNA from the flash-dried leaves using the DNeasy Plant Mini Kit (QIAGEN, Germantown, MD, USA), and amplified nine microsatellite loci chosen from those developed by Yang et al. (2005) and Gallagher et al. (2004) based on ease of amplification and the presence of sufficient polymorphism (Supplementary Table S1). Each of the $10 \mu \mathrm{L}$ microsatellite PCR reactions started with a 5-min denaturation at $94^{\circ} \mathrm{C}$, followed by 40 cycles of $94^{\circ}$ $\mathrm{C}$ for $45 \mathrm{~s}, 55-61^{\circ} \mathrm{C}$ for $30 \mathrm{~s}$ and $72{ }^{\circ} \mathrm{C}$ for $30 \mathrm{~s}$ followed by a final 10 -min extension at $72{ }^{\circ} \mathrm{C}$. Fragment analysis used the GenomeLab GeXP Genetic Analysis System (Beckman Coulter, Indianapolis, IN, USA) and CEQ 8000 Genetic Analysis System (Beckman Coulter, Indianapolis, IN, USA). Microsatellite sizes were binned by hand. The size range of the alleles at each locus ranged 10-58 base pairs (bp) and averaged 40.7 bp (Supplementary Table S2).

\section{Analysis of hierarchical phenotypic and genetic structure}

By fitting random-effects hierarchical linear models to four floral traits, we estimated the variance: among outcrops, among plots within outcrops, among flowers nested within plots and among replicate measurements within flowers. The latter provides an estimate of measurement error, which was very low for all traits $(<1.6 \%$ of total variation for all traits, Table 2). Using the lmer function in the lme4 package (v. 1.2-12, Bates et al. 2015) in the R statistical environment (v.3.1.2, R Development Core Team 2017), we estimated the random variance components with restricted maximum likelihood (REML) and used likelihood ratio

Table 2 Partitioning of variation in floral morphology, allele frequencies at nine polymorphic microsatellite loci, and assignment probabilities from a Bayesian InStruct clustering analysis among hierarchical samples of Aquilegia canadensis

\begin{tabular}{lllll}
\hline Trait / marker & $\begin{array}{l}\text { Among } \\
\text { outcrops }\end{array}$ & $\begin{array}{l}\text { Among } \\
\text { plots } \\
\text { within } \\
\text { outcrops }\end{array}$ & $\begin{array}{l}\text { Among } \\
\text { plants } \\
\text { within } \\
\text { plots }\end{array}$ & $\begin{array}{l}\text { Measurement } \\
\text { error }\end{array}$ \\
\hline Herkogamy & 1.89 & 0.74 & $96.47^{*}$ & 0.89 \\
Sepal length & 0.00 & $7.88^{*}$ & $90.54^{*}$ & 1.58 \\
Spur length & 7.48 & 1.99 & $89.82^{*}$ & 0.70 \\
Pistil length & 2.27 & 2.84 & $93.86^{*}$ & 1.02 \\
Microsatellite & $1.09 *$ & $2.52^{*}$ & $96.39^{*}$ & - \\
Assignment prob & $12.87 * *$ & $8.05^{*}$ & 79.08 & - \\
\hline
\end{tabular}

Values are the percentage of variation at each hierarchical level. Plants were sampled from two plots ( 20 plants per plot) within each of seven outcrops. Each flower was measured twice to estimate measurement error. Statistical significance of the variance components is indicated as: $* P<0.05, * * P<0.001$. Note that the variance among plants within plots for the assignment probability cannot be tested for significance because individuals served as the basic unit of analysis 
tests to evaluate whether individual variance components differed from zero. Because floral traits often vary with flower age and inflorescence position, we included number of dehisced anthers and floral rank as fixed-effect covariates. Following Zuur et al. (2009), we evaluated these covariates using likelihood ratio tests with models fit using maximum likelihood (Supplementary Table S3). Age was always significant, but rank never was. Therefore, we report analyses of random variance components controlling only for flower age below. Visual inspection of residuals did not reveal violations of homoscedasticity or normality.

We partitioned the variance in microsatellite allele frequencies using hierarchical analysis of molecular variance (AMOVA) implemented in Arlequin (v. 3.5, Excoffier and Lischer 2010) with outcrop, plot within outcrop and individual within plot as hierarchical levels. We then estimated genetic variances attributable to each hierarchical level, which sum to total genetic variance. Arlequin was also used to estimate hierarchical $F$-statistics $\left(F_{\mathrm{IT}}, F_{\mathrm{CT}}, F_{\mathrm{SC}}\right.$ and $F_{\mathrm{IS}}$ where $\mathrm{I}$ is individual, $\mathrm{S}$ plot, $\mathrm{C}$ outcrop and $\mathrm{T}$ total) along with their $95 \%$ confidence intervals derived from 20000 bootstraps.

\section{Analysis of isolation by distance}

At drift-gene flow equilibrium, pairwise genetic distance between samples should correlate positively with geographic distance between sampling locations (isolation-bydistance, IBD). Local selection may perturb the pattern of IBD for floral traits. We used Mantel's tests (along with a permutation test to estimate $P$ values), based on Spearman's rank correlations, implemented in the $\mathrm{R}$ package vegan (version 2.4-0; Oksanen et al. 2016) to test IBD. As highlighted by Legendre and Fortin (2010), the Mantel test assumes a linear relationship between distances in the two matrices being evaluated. This assumption can be relaxed by using the Spearman rank correlation instead of the Pearson correlation. Pairwise genetic distance was calculated as linearized $F_{\mathrm{ST}}$ between plots $\left(F_{\mathrm{ST}} /\left(1-F_{\mathrm{ST}}\right)\right)$ estimated using Arlequin v.3.5 (Excoffier and Lischer 2010). Pairwise geographic distances between plots (in $\mathrm{m}$ ) were calculated as two-dimensional Euclidean distances from UTM coordinates recorded in the center of each plot. Pairwise distances for floral morphology were calculated as the 2-dimensional Euclidean distances between plot centroids on the first two principal components (PCs) of the individual-level correlation matrix between the four floral traits. The first two PCs together accounted for $78.0 \%$ of the total floral variation. Significance was evaluated by comparing estimated correlation coefficients $(r)$ to null distributions generated by 10000 permutations of the data. We also tested for a correlation between the pairwise floral distances and microsatellite genetic distances to evaluate whether similar processes were influencing the spatial patterns of both classes of variation.

\section{Analysis of genetic structure}

We conducted a Bayesian cluster analysis using InStruct (Gao et al. 2007) to test for genetic structure independent of our hierarchical sampling design. InStruct implements the Markov Chain Monte Carlo algorithm for generalized Bayesian clustering. It assumes markers are unlinked but does not require Hardy-Weinberg equilibrium. We simulated 200,000 MCMC iterations with 100,000 burn-in steps for each chain, and 10 replicate chains for each of the possible number of genetic clusters, $K$ (up to a maximum of 14 , the number of plots). We used the default parameters of the admixture model (Mode 1), which infers population structure only. We based our choice of optimal $K$ on Delta $K$, which captures the best representation of higher order structure (Evanno et al. 2005). The assignment probabilities for each individual to the most likely clusters, for each optimal $K$ model, were averaged over replicate chains using the algorithm implemented in CLUMPP (Jakobsson and Rosenberg 2007).

To complement the AMOVA described above, we tested for spatial variation in InStruct cluster assignment probabilities as follows. First, we fit individual cluster assignment probabilities (probability of assignment to cluster 1 of the two clusters detected, see "Results" section) to a hierarchical random-effects linear model with outcrops and plots within outcrops as levels (as above). Because the response variable (assignment to cluster 1) is a proportion, we applied the logit transformation in the car package for $\mathrm{R}$ (v. 2.1-4, Fox and Weisberg 2011). Next, we tested for IBD in assignment probabilities among plots (also as above) using pairwise $t$-values calculated from logit-transformed assignment probabilities as a measure of genetic distance between plots.

We also compared the InStruct results to those obtained from the program TESS3, which implements admixture models while explicitly accounting for geographic information in estimating population structure. The algorithm was run using the R package "tess3r" (Caye et al. 2016). Because we only had plot coordinates, individual coordinates were generated using the "Generate Spatial Coordinates" option in TESS (v. 2.3.1, Chen et al. 2007). We used the default settings along with the Projected Least Squares algorithm to estimate ancestry matrices. The choice of optimal $\mathrm{K}$ was chosen where the plot of cross-validation score reached a plateau (Caye et al. 2016). The crossvalidation score is the root mean-squared errors computed on a subset of loci. 


\section{Fine-scale genetic structure}

To address the possibility of neutral genetic structure at scales smaller than between sampling plots $(<30 \mathrm{~m})$, we compared genetic relatedness between pairs of plants that were nearest neighbors and pairs that were not nearest neighbors. We sampled the nearest co-flowering conspecific to 45 randomly-sampled focal plants on one rock outcrop (QRL1, sampled independently of the 40 sampled in the two plots for hierarchical analyses, Table 1). Co-flowering plants both had $\geq 1$ flower with 11-29 dehisced anthers on at least 1 day. The distance from each focal plant to its neighbor was measured to $1 \mathrm{~cm}$. This yielded 73 plants in all, rather than 90 (i.e., $45 \times 2$ ), because 15 plants had nearest neighbors that were other focal plants and some focal plants shared the same nearest neighbor. We assayed these 73 plants for 10 microsatellite loci (the nine used above plus an additional locus, Supplementary Table S1) and obtained genotypes for $90.3 \%$ of all locus by plant combinations.

Using the program Mark (v. 3.1, Ritland 2006), we estimated pairwise relatedness $(r)$ between all 73 plants. Several estimators are available in Mark 3.1. We present the results of four relatedness estimators: Queller and Goodnight $1989=$ QG; Ritland $1996=\mathrm{R}$; Lynch and Ritland $1999=\mathrm{LR}$; Wang $2002=\mathrm{W}$. Each estimator performs best for different population compositions (i.e., the level of marker polymorphism and genetic relations among samples, e.g., parent-offspring, full-sib, half-sib). Because the genetic relationship among samples was unknown, and likely to be quite variable, we had no a priori reason to assume one method would outperform the others. For each estimator, this yielded a matrix of nonindependent $r$ values, so we used permutations tests, performed with $\mathrm{R}$, to assess whether the average $r$ of nearest neighbors was greater than that of non-nearest neighbors (derived from all the pairwise comparisons between plants that were not nearest neighbors). Permutation tests (10,000 permutation) also evaluated whether $r$ decreased with increasing distance between nearest neighbors.

\section{Results}

\section{Hierarchical variation for floral traits and genetic diversity}

Almost all the variance of each floral trait (herkogamy, sepal length, spur length and pistil length) after controlling for floral age was partitioned among plants (flowers) within plots (Table 2, Supplementary Figure S2). Sepal length varied somewhat among plots within outcrops $(7.88 \%$ of total variance), but all other variance components for all other traits were very low (typically $<5 \%$ ) and not different from zero.

Genetic diversity measured as expected heterozygosity for each plot ranged 0.61-0.72 (Supplementary Table S4). Similar to the spatial distribution of floral variation, the analysis of molecular variance indicated that most genetic variation at microsatellite loci was partitioned among plants within plots ( $>96 \%$, Table 2). Variance components among outcrops and among plots within outcrops although significant were very small (1.09 and $2.52 \%$, respectively). All estimates of hierarchical $F$ statistics were greater than zero but components among outcrops and between plots within outcrops were small $\left(F_{\mathrm{IS}}=0.27\right.$ [95\% CI $\left.=0.20-0.36\right], F_{\mathrm{SC}}$ $=0.017$ [0.0092-0.024], $F_{\mathrm{CT}}=0.013$ [0.0074-0.020], $F_{\mathrm{IT}}$ $=0.29[0.22-0.39]$; where $\mathrm{I}=$ individuals, $\mathrm{S}=$ plots, $\mathrm{C}=$ outcrops, $\mathrm{T}=$ total).

\section{Geographic pattern of floral and genetic differentiation}

We did not detect isolation-by-distance (IBD) for either floral morphology or for microsatellite polymorphisms (Fig. 2). Although both correlations were positive, neither was close to significant (floral morphology: $r=+0.00101$, $\left.P_{\text {perm }} \sim 0.46 ; F_{\mathrm{ST}}: r=+0.0297, P_{\text {perm }} \sim 0.37\right)$. Pairwise $F_{\mathrm{ST}}$ estimates among plots were all low $(<0.08)$ and only half $(52 \%)$ were greater than zero after Dunn-Sidak corrections
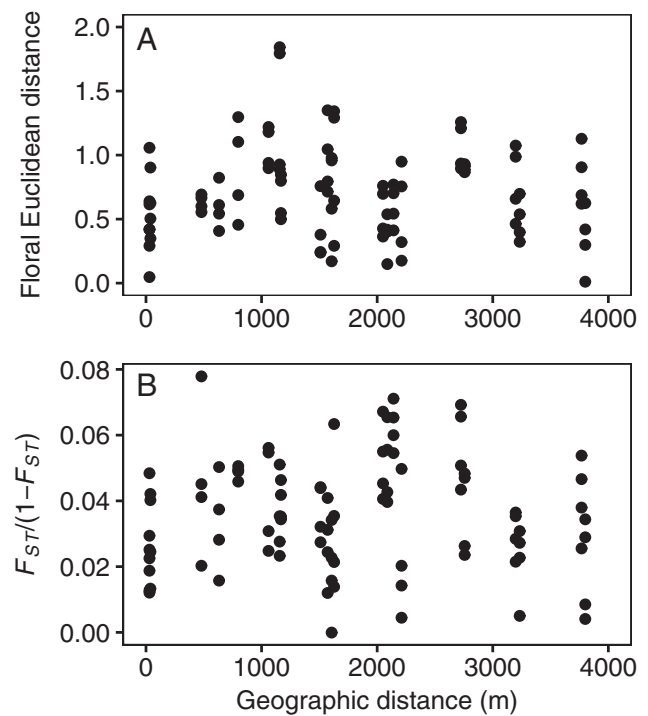

Fig. 2 Relations between geographic distance and morphological (a) and neutral genetic (b) distance (IBD) among samples of Aquilegia canadensis from pairs of plots (20 plants per plot) within each of seven outcrops. Each point represents a pairwise comparison between plots. a Shows pairwise distances for floral morphology measured as the Euclidean distance between plot centroids for principal components of floral traits $\left(r=+0.00101, P_{\text {perm }} \sim 0.46\right)$. b Shows linearized pairwise genetic distance (linearized $F_{\mathrm{ST}}$ ) for microsatellite polymorphisms $(r$ $\left.=+0.0297, P_{\text {perm }} \sim 0.37\right)$ 


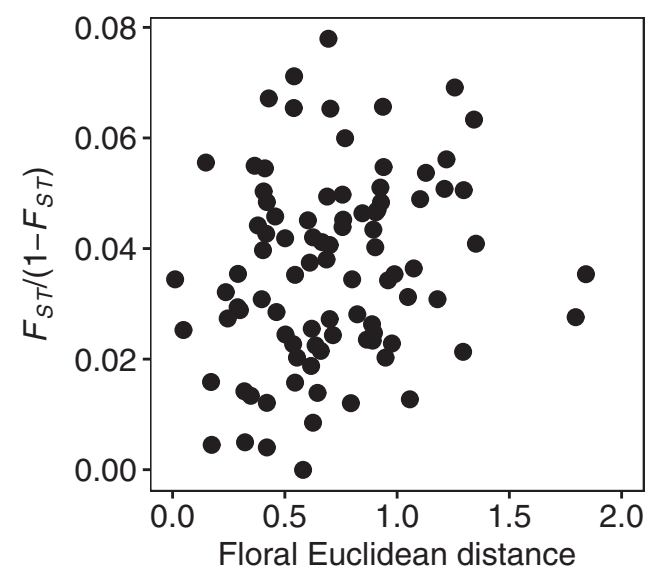

Fig. 3 Correlation of pairwise differences between plots in floral morphology and microsatellite allele frequencies for Aquilegia canadensis sampled from pairs of plots (20 plants per plot) within each of seven outcrops $\left(r=+0.221, P_{\text {perm }} \sim 0.099\right)$. See Fig. 2 for details

for multiple comparisons (Supplementary Table S5). Pairwise differentiation in floral morphology and pairwise $F_{\mathrm{ST}}$ correlated positively though not quite significantly $(r=+$ 0.221, $P_{\text {perm }} \sim 0.099$, Fig. 3).

\section{Structure analysis}

InStruct analysis suggested two clusters based on the $\Delta K$ criterion (Fig. 1 left panel; Supplementary Figure S3). Most individuals were highly admixed between these clusters. No individual had an estimated assignment probability of zero or one, and $60.4 \%$ of individuals had an assignment probability between 0.2 and 0.8 for either cluster. Similar to AMOVA results, most variation in cluster assignment probability was partitioned among individuals (79.1\%) within plots, with relatively little among plots within outcrops $(8.0 \%)$ and among outcrops (12.9\%), although all components of variance were statistically significant (Table 2). Plots closer geographically were not more likely to be assigned to the same cluster (IBD $r=+0.022, P_{\text {perm }} \sim$ $0.38)$.

TESS3 analysis detected 10 clusters (Supplementary Figure S4) and, again, individuals were highly admixed. Nearly all individuals $(98.2 \%)$ had a maximum assignment probability of 0.2-0.8 (Supplementary Figure S4).

\section{Fine-scale neutral genetic structure}

All four measures of pairwise genetic relatedness between individuals $(r)$ correlated strongly (Pearson correlation coefficient $=+0.63-0.87$, all $P_{\text {perm }}<0.0001$ ). Although $r$ between nearest neighbors $\left(r_{\mathrm{NN}}\right)$ was low (Table 3, mean $\left.r_{\mathrm{NN}}=+0.048\right)$, it was higher than $r$ between plants that were not nearest neighbors (mean $r_{\text {non-NN }}=-0.0035$, all $\left.P_{\text {perm }}<0.0001\right)$. The distance between nearest neighbors
Table 3 Pairwise relatedness of nearest neighbor Aquilegia canadensis compared to randomly paired non-neighbors based on four measures of genetic relatedness

\begin{tabular}{llll}
\hline Relatedness estimator & $r_{\mathrm{NN}}$ & $r_{\text {non-NN }}$ & $P_{\text {perm }}$ \\
\hline QG & $0.049 \pm 0.160$ & $-0.006 \pm 0.105$ & 0.0002 \\
R & $0.089 \pm 0.111$ & $0.044 \pm 0.042$ & $<0.0001$ \\
LR & $0.038 \pm 0.113$ & $-0.009 \pm 0.047$ & $<0.0001$ \\
W & $0.016 \pm 0.160$ & $-0.044 \pm 0.092$ & $<0.0001$
\end{tabular}

Measures of relatedness: Queller and Goodnight (1989)=QG; Ritland $(1996)=\mathrm{R}$; Lynch and Ritland $(1999)=\mathrm{LR}$; Wang $(2002)=\mathrm{W}$. Coefficients of relatedness $(r \pm 1 \mathrm{SD})$ are in columns for nearest neighbors $\left(r_{\mathrm{NN}}\right)$ and non-nearest neighbors $\left(r_{\text {non-NN }}\right)$. Permutation tests ( $P_{\text {perm }}$ based on 10000 permutations of the data) assessed if $r_{\mathrm{NN}}=r_{\text {non- }}$ NN

ranged $6-620 \mathrm{~cm}$, averaged $( \pm 1 \mathrm{SD}) 86.2 \pm 113.5 \mathrm{~cm}$ and correlated negatively with $r_{\mathrm{NN}}$ (Pearson correlation coefficient $=-0.20$ to -0.15 ). However, only the correlation based on the Ritland (1996) estimator was statistically significant (Pearson correlation coefficient $=-0.20, P=$ 0.038).

\section{Discussion}

\section{Weak genetic structure for microsatellites and floral traits}

Owing to the consequent reductions in effective population size and gene flow, predominant self-fertilization should encourage the development of fine-scale spatial genetic structure (SGS). Populations of A. canadensis in eastern Ontario self-fertilize $75 \%$ of seed, on average, and results from previous manipulative experiments are consistent with the occurrence of SGS within populations (Griffin and Eckert 2003; Herlihy and Eckert 2004). In contrast, the direct analyses of population structure we report here involving both putatively neutral microsatellite polymorphisms and heritable variation in floral traits detected only very weak SGS. We detected some variance among outcrops and between plots within outcrops for microsatellite allele frequencies and InStruct cluster assignment probabilities, but these components of variance were small compared to variance among individuals within plots. Furthermore, differentiation between plots was not related to spatial proximity. There was no isolation-by-distance among plots for floral morphology, or microsatellite allele frequencies. Only at much finer scales, within plots, did we detect distance-dependent SGS, although this too was weak. Thus, counter to theoretical expectations, high rates of selffertilization in A. canadensis populations (Herlihy and Eckert 2004) do not result in strong SGS within or among populations. 
Analyses of molecular and phenotypic variance revealed that almost all the variation occurs among plants within plots of $A$. canadensis rather than among outcrops or plots within outcrops. This is not because A. canadensis has exceptionally high variation within plots, rather variation at higher levels is simply low. Within plot microsatellite polymorphism measured as alleles per locus and expected heterozygosity averaged 7.40 and 0.670 , respectively (Supplementary Table S4). This level of genetic diversity is similar to values reported within populations of predominantly outcrossing plants (Charlesworth 2003), but slightly lower than that reported in related species $A$. coerulea (8.94 and 0.74, respectively; Brunet et al. 2012). Similarly, within-plot phenotypic variation for the three structural floral traits as measured by the between-plant coefficient of variation (Supplementary Table S6, median $\mathrm{CV}$ : pistil length $=11 \%$, sepal length $=11 \%$, spur length $=$ $8 \%$ ) was on par with Cresswell's (1998) meta-analysis of within-population phenotypic variation from 151 largely outcrossing plant species for attractive traits (median $\mathrm{CV}=$ $16 \%$, comparable to sepal length), sexual organ dimensions (11\%, comparable to pistil length) and traits involved in matching between flowers and pollen vectors (13\%, comparable to spur length); as well as Wolfe and Krstolic's (1999) analysis of petal length in 16 outcrossing species with radially symmetric flowers (12\%). Overall, the levels of phenotypic and genetic diversity that we documented within A. canadensis populations are on par with estimates of other predominantly outcrossing species, suggesting that the low amount of variance attributed to higher levels was not caused by exceptionally high variance among individuals.

\section{Weak fine-scale genetic structure}

At the smallest scale of our study $(<10 \mathrm{~m})$ we found evidence of fine-scale structure. Estimates of relatedness $(r)$ were generally low $(r<0.10)$, but were higher between nearest neighbors than randomly paired non-neighbors. Additionally, $r$ declined with increasing distance between neighbors. Distance-dependent relatedness is commonly detected in plant populations, and is usually viewed as arising from localized seed dispersal (Vekemans and Hardy 2004), which is likely in A. canadensis (see below). Such fine-scale SGS may be responsible for previous experimental observations that moving plants over the scale of 2-10 $\mathrm{m}$ within populations resulted in lower biparental inbreeding (Griffin and Eckert 2003) and that flowers rendered phenotypically female by emasculation still engaged in significant apparent selfing (Herlihy and Eckert 2004). However, Herlihy and Eckert's (2004) estimates of $r$ between outcross mates based on the amount of apparent selfing by emasculated flower were much higher $\left(m_{\mathrm{b}} \sim+\right.$
0.50) than $r$ between nearest neighbors measured here (mean $\left.r_{\mathrm{NN}}=+0.048\right)$.

Under the most localized pollinator movements, $r_{\mathrm{NN}}$ should be the upper limit of the average genotypic relatedness among non-self mates $\left(m_{b}\right)$, unless there are factors, in addition to spatial proximity, which promote assortative mating among relatives. One likely possibility is flowering phenology. Initiation of flowering is usually heritable in plant populations (Geber and Griffen 2003), so it is conceivable that $r$ may correlate with overlap in phenology (Weis and Kossler 2004). Within the populations of $A$. canadensis in our study area, individuals initiate flowering over a 2-3 wk period (Kliber and Eckert 2004 Digital Appendix A). Although the sequential production of several flowers per individual may increase phenological overlap among individuals that initiated flowering at different times, first flowers escape herbivory more frequently than later flowers (Kliber and Eckert 2004), which likely reduces overlap and strengthens phenological assortative mating. Further work is needed to establish to what extent $r$ correlates with an overlap in phenology.

\section{Inbreeding depression may erode spatial genetic structure by enhancing effective gene flow}

Spatial genetic structure is influenced by the balance between genetic drift promoting spatial differentiation and gene flow that erodes it. Self-fertilization reduces $N_{\mathrm{e}}$ and opportunities for gene flow by pollen thereby hastening differentiation among populations. Outcrossing, in contrast, erodes SGS across populations, particularly if gene flow is more extensive via pollen than via seed, as generally expected (Petit et al. 2005; Ellstrand 2014). In partially selfing populations, the net genetic structure exhibited by mature plants will depend on the relative production and survival of selfed vs. outcrossed progeny (Nakanishi et al. 2015). On the basis of the progeny array analysis conducted at the seed stage, A. canadensis self-fertilizes $\sim 75 \%$ of seed on average (Eckert and Herlihy 2004), yet the inbreeding coefficient $(F)$ of adult plants is typically much lower than expected from the selfing rate (though still $>0$, Table S2), implying that selfed offspring survive to reproduce $<1 / 10$ as frequently as outcrossed offspring (Herlihy and Eckert 2004, 2005). Strong inbreeding depression will tend to make the SGS of a partially selfing population resemble that of a more outcrossing population because offspring produced by outcrossing become more frequent among surviving and reproducing individuals. However, empirical evidence supporting this expectation is scant because few studies have contrasted SGS for different traits/loci in species where the degree of self-fertilization and the strength of inbreeding depression are known (but see Brunet et al. 2012). 
The results of our study are in line with evidence that inbreeding depression plays an important role in modifying population structure in other species of Aquilegia. Brunet et al. (2012) suggest that selection against selfed progeny during early life history stages in A. coerulea may explain the lack of correlation between estimates of selfing based on analysis of progeny arrays assayed as seed and the inbreeding coefficient $(F)$ of mature plants, as well as the significant gene flow and isolation by distance among populations detected over a scale of hundreds of kilometers. Yang and Hodges (2010) documented a progressive decrease in $F$ through the life cycle in A. formosa and $A$. pubescens consistent with higher mortality of selfed than outcrossed offspring. Both species also exhibit extensive gene flow and isolation by distance among populations separated by tens of kilometers (Noutsos et al. 2014). Similar results have also been documented in other species. In a study of viability selection at different life history stages, Hufford and Hamrick (2003) found strong viability selection against inbred individuals acting between mature seed and seedling stages (Hufford and Hamrick 2003). Thus, the inbreeding coefficient of the population at reproduction was lower than expected based on selfing rates. Comparing SGS along with the inbreeding coefficient $(F)$ at different life history stages would confirm that selection against inbred individuals prior to reproduction causes the mating population to resemble a more outcrossing population (Tonsor et al. 1993; Hufford and Hamrick 2003).

\section{Ecological factors may also reduce SGS}

Ecological factors that determine adult plant densities, and seed dispersal will influence SGS. The quality and physical configuration of habitat can influence plant density, a major component of effective population size, which is expected to determine the strength of genetic drift and thus SGS at the population level (Vekemans and Hardy 2004). Density can also influence fine-scale SGS within local populations (Cruse-Sanders and Hamrick 2004). A low density of reproductive plants with restricted seed shadows can generate fine-scale structure because individuals will tend to have sibs as nearest neighbors. High density with broad overlap in seed shadows will, in contrast, lead to weak SGS as seed families intermingle spatially. Although A. canadensis is patchily distributed on the rock outcrops it inhabits, plants are typically at high density. Nearest neighbor distances, which correlate strongly with density in eastern Ontario A. canadensis populations (Herlihy and Eckert 2004 ), were $<1 \mathrm{~m}$ on average. The density of reproductive plants within populations in our study area used by Herlihy and Eckert (2004) ranged 1.3-40.7 plants $/ \mathrm{m}^{2}$ and averaged
6.9 plants $/ \mathrm{m}^{2}$. Seeds of A. canadensis have no traits that obviously enhance dispersal as they simply fall or are shaken from dehiscent follicles held $<0.5 \mathrm{~m}$ above the ground. However, seed movement mediated by rapid rain runoff in the open rocky habitats where the species occurs may transport seeds over the scale of meters. Taken together, this suggests considerable mixing of seed shadows among neighboring plants and hence weak fine-scale SGS within populations.

Pollen movement during outcrossing may further erode SGS at a broader range of spatial scales depending on the animals facilitating pollen transfer. Outcross pollination in A. canadensis is mediated by bumble bees (Bombus spp.) and ruby-throated hummingbirds (Archilochus colubris, Macior 1996; C.G. Eckert, unpublished data), both of which, but especially the latter, may move pollen long distances, especially during spring migration (Herlihy and Eckert 2005). Pollination by strong-flying pollinators such as bumble bees, hummingbirds and hawkmoths is associated with extensive gene flow over large spatial scales in other species of Aquilegia (Brunet et al. 2012; Noutsos et al. 2014). Bumble bee and hummingbird pollinators could easily traverse the distance between the seven outcrops surveyed in this study, thus contributing to gene flow and reducing SGS among populations.

\section{No spatial differentiation of floral morphology}

Aquilegia includes $>70$ species with a pan-temperate distribution and has been cited as one of the most dramatic examples of differentiation in floral morphology among species in response to selection imposed by diverse pollinators (Whittall and Hodges 2007; Hodges and Derieg 2009). Yet floral differentiation within species and the underlying evolutionary mechanisms have rarely been studied (Thairu and Brunet 2015).

Local differentiation in floral morphology at the spatial scales examined here was very weak and on par with that observed for neutral microsatellite polymorphisms. This suggests that the floral variation we detected may be selectively neutral in the area we studied. In contrast, Herlihy and Eckert (2005) found strong phenotypic differentiation in floral morphology among populations of $A$. canadensis across very large spatial scales (1000's of km). It is possible that phenotypic selection is strong and variable among populations but that differentiation of floral traits has not occurred due to insufficient heritable variation. While studies on A. coerulea demonstrating significant phenotypic plasticity of floral traits (including pistil length and herkogamy; Brunet et al. 2012; Van Etten and Brunet 2013) suggest that the phenotypic variation we observed could arise from small-scale environmental variation, previous 
studies on $A$. canadensis in our eastern Ontario study area indicate significant heritable variation in the floral traits we measured. Herlihy and Eckert (2007) estimated mean broad-sense heritabilities from among family phenotypic variation in a common environment of 0.46 for herkogamy, 0.20 for pistil length and 0.24 for spur length for three eastern Ontario populations including QLL3 studied here. Regression between progeny phenotypes measured in the glasshouse and maternal phenotypes measured in the field were also significant for herkogamy (mean standardized regression coefficient $=+0.43$ ) and pistil length $(+0.32)$.

Another interpretation is that selection is strong but favors a common floral phenotype across the region we studied. Although we cannot confidently reject this hypothesis, it too is inconsistent with the occurrence of substantial heritable variation in floral morphology within A. canadensis populations in our study area (Herlihy and Eckert 2007). Moreover, preliminary analysis of phenotypic selection detected only weak and predominantly nonsignificant selection on the floral traits we measured in eastern Ontario populations of A. canadensis (C.G. Eckert, unpublished data). Castellanos et al. (2011) also detected only weak and usually nonsignificant selection on floral traits within 13 populations of $A$. pyrenaica and $A$. vulgaris in Europe. In light of the previous studies of $A$. canadensis in eastern Ontario, our results suggest that the pattern of variation in floral morphology at the local spatial scale examined here seems to be dictated by a simple interplay between drift and extensive gene flow via outcrossing.

\section{Data archiving}

Nuclear microsatellite genotypes are available in the DRYAD repository: https://doi.org/10.5061/dryad.588d7. Floral morphological data for each population are available at DRYAD: https://doi.org/10.5061/dryad.588d7.

Acknowledgements We thank the Queen's University Biological Station for logistic support in the field, Barbara Mable for comments on the manuscript, and the Natural Sciences and Engineering Research Council of Canada (NSERC) for an Undergraduate Summer Research Award to LZ and a Discovery Grant to CGE.

\section{Compliance with ethical standards}

Conflict of interest The authors declare that they have no conflict of interest.

\section{References}

Bates D, Maechler M, Bolker B, Walker S (2015) Fitting linear mixedeffects models using lme4. J Stat Soft 67:1-48

Brommer JE (2011) Whither $P_{S T}$ ? The approximation of $Q_{S T}$ by $P_{S T}$ in evolutionary and conservation biology. $J$ Evol Biol 24:1160-1168
Brunet J, Larson-Rabin Z, Stewart CM (2012) The distribution of genetic diversity within and among populations of the Rocky Mountain columbine: the impact of gene flow, pollinators and mating system. Int J Plant Sci 173:484-494

Castellanos MC, Alcantara JM, Rey PJ, Bastida JM (2011) Intrapopulation comparison of vegetative and floral trait heritabilities estimated from molecular markers in wild Aquilegia populations. Mol Ecol 20:3513-3524

Caye K, Deist TM, Martins H, Michel O, François O (2016) TESS3: fast inference of spatial population structure and genome scans for selection. Mol Ecol Res 16:540-548

Charlesworth D (2003) Effects of inbreeding on the genetic diversity of populations. Philos Trans Roy Soc Lond B 358:1051-1070

Chen C, Durand E, Forbes F, François O (2007) Bayesian clustering algorithms ascertaining spatial population structure: a new computer program and a comparison study. Mol Ecol Notes 7:747-756

Cresswell JE (1998) Stabilizing selection and the structural variability of flowers within species. Ann Bot 81:463-473

Cruse-Sanders JM, Hamrick JL (2004) Spatial and genetic structure within populations of wild American ginseng (Panax quinquefolius L., Araliaceae). J Hered 95:309-321

DeFaveri J, Merilä J (2013) Evidence for adaptive phenotypic differentiation in Baltic Sea sticklebacks. J Evol Biol 26:1700-1715

Eckert CG, Herlihy CR (2004) Using a cost-benefit approach to understand the evolution of self-fertilization in plants: the perplexing case of Aquilegia canadensis (Ranunculaceae). Plant Species Biol 19:159-173

Ellstrand NC (2014) Is gene flow the most important evolutionary force in plants? Am J Bot 101:737-753

Evanno G, Regnaut S, Goudet J (2005) Detecting the number of clusters of individuals using the software STRUCTURE: a simulation study. Mol Ecol 14:2611-2620

Excoffier L, Lischer HE (2010) Arlequin suite ver. 3.5: a new series of programs to perform population genetics analyses under Linux and Windows. Mol Ecol Res 10:564-567

Fox J, Weisberg S (2011) An R companion to applied regression, 2nd edn. Sage, Thousand Oaks

Gallagher KG, Milligan BG, White PS (2004) Isolation and characterization of microsatellite DNA loci in Aquilegia spp. Mol Ecol Notes 4:686-688

Gao H, Williamson S, Bustamante CD (2007) A Markov Chain Monte Carlo approach for joint inference of population structure and inbreeding rates from multilocus genotype data. Genetics 176:1635-1651

Geber MA, Griffen LR (2003) Inheritance and natural selection on functional traits. Int J Plant Sci 164:S21-S42

Glémin S, Bazin E, Charlesworth D (2006) Impact of mating systems on patterns of sequence polymorphism in flowering plants. Proc R Soc B 273:3011-3019

Griffin CAM, Eckert CG (2003) Experimental analysis of biparental inbreeding in a self-fertilizing plant. Evolution 57:1513-1519

Griffin SR, Mavraganis K, Eckert CG (2000) Experimental analysis of protogyny in Aquilegia canadensis (Ranunculaceae). Am J Bot 87:1246-1256

Hamrick JL, Godt MJW (1996) Effects of life-history traits on genetic diversity in plant species. Philos Trans R Soc Lond Biol Sci 351:1291-1298

Herlihy CR, Eckert CG (2004) Experimental dissection of inbreeding and its adaptive significance in a flowering plant, Aquilegia canadensis (Ranunculaceae). Evolution 58:2693-2703

Herlihy CR, Eckert CG (2005) Evolution of self-fertilization at geographic range margins? A comparison of demographic, floral, and mating system variable in central vs. peripheral populations of Aquilegia canadensis (Ranunculaceae). Am J Bot 92:744-751 
Herlihy CR, Eckert CG (2007) Evolutionary analysis of a key floral trait in Aquilegia canadensis (Ranunculaceae): Genetic variation in herkogamy and its effect on the mating system. Evolution 61:1661-1674

Hufford KM, Hamrick JL (2003) Viability selection at three early life stages of the tropical tree, Platypodium elegans (Fabaceae, Papilionoideae). Evolution 57:518-526

Hodges SA, Derieg NJ (2009) Adaptive radiations: from field to genomic studies. Proc Natl Acad Sci USA 106:9947-9954

Ingvarsson PK (2002) A metapopulation perspective on genetic diversity and differentiation in partially self-fertilizing plants. Evolution 56:2368-2373

Jakobsson M, Rosenberg NA (2007) CLUMPP: a cluster matching and permutation program for dealing with label switching and multimodality in analysis of population structure. Bioinformatics 23:1801-1806

Kliber A, Eckert CG (2004) Sequential decline in allocation among flowers within inflorescences: proximate mechanisms and adaptive significance. Ecology 85:1675-1687

Legendre P, Fortin MJ (2010) Comparison of the Mantel test and alternative approaches for detecting complex multivariate relationships in the spatial analysis of genetic data. Mol Ecol Res 10:831-844

Loveless MD, Hamrick JL (1984) Ecological determinants of genetic structure in plant populations. Ann Rev Ecol Syst 15:65-95

Lynch M, Ritland K (1999) Estimation of pairwise relatedness with molecular markers. Genetics 152:1753-1766

Macior LW (1996) Foraging behavior of Bombus (Hymenoptera: Apidae) in relation to Aquilegia pollination. Am $\mathbf{J}$ Bot 53:302-309

Nakanishi A, Yoshimaru H, Tomaru N, Miura M, Manabe T, Yamamoto S (2015) Inbreeding depression at the sapling stage and its genetic consequences in a population of the outcrossing dominant tree species, Castanposis sieboldii. Tree Gen Genomes 11:62-71

Noutsos C, Borevitz JO, Hodges SA (2014) Gene flow between nascent species: geographic, genotypic and phenotypic differentiation within and between Aquilegia formosa and A. pubescens. Mol Ecol 23:5589-5598

Petit RJ, Duminil J, Fineschi S, Hampe A, Salvini D, Vendramin GG (2005) Comparative organization of chloroplast, mitochondrial and nuclear diversity in plant populations. Mol Ecol 14:689-701

Oksanen J, Blanchet FG, Friendly M, Kindt R, Legendre P, McGlinn Dan, Minchin PR, O'Hara RB, Simpson GL, Solymos P, Stevens MHH, Szoecs E, Wagner H (2016). vegan: community ecology package. R package version 2.0-2. https://CRAN.R-project.org/pa ckage $=$ vegan

Queller DC, Goodnight KF (1989) Estimating relatedness using genetic markers. Evolution 43:258-275
R Development Core Team (2017) R: A language and environment for statistical computing. R Foundation for Statistical Computing, Vienna, Austria

Ritland K (1996) Estimators for pairwise relatedness and individual inbreeding coefficients. Gen Res 67:175-185

Ritland K (2006) Mark v. 3.1-marker inferred relatedness and quantitative inheritance program. University of British Columbia, Vancouver

Schemske DW, Bierzychudek P (2007) Spatial differentiation for flower color in the desert annual Linanthus parryae: was Wright right? Evolution 61:2528-2543

Schoville SD, Bonin A, François O, Lobreaux S, Melodelima C, Manel S (2012) Adaptive genetic variation on the landscape: methods and cases. Ann Rev Ecol Evol Syst 43:23-43

Sexton JP, Hangartner SB, Hoffman AA (2013) Genetic isolation by environment or distance: which pattern of gene flow is most common? Evolution 68:1-15

Thairu MW, Brunet J (2015) The role of pollinators in maintaining variation in flower color in the Rocky Mountain columbine, Aquilegia coerulea. Ann Bot 115:971-979

Tonsor SJ, Kalisz S, Fisher J, Holtsford TP (1993) A life-history based study of population genetic structure: seed bank to adults in Plantago lanceolata. Evolution 47:833-843

Van Etten ML, Brunet J (2013) The impact of global warming on floral traits that affect the selfing rate in a high-altitude plant. Int $\mathrm{J}$ Plant Sci 174:1099-1108

Vekemans X, Hardy OJ (2004) New insights from fine-scale spatial genetic structure analyses in plant populations. Mol Ecol 13:921-935

Wang J (2002) An estimator for pairwise relatedness using molecular markers. Genetics 160:1203-1215

Weis AE, Kossler TM (2004) Genetic variation in flowering time induces phenological assortative mating: quantitative genetic methods applied to Brassica rapa. Am J Bot 91:825-836

Whittall JB, Hodges SA (2007) Pollinator shifts drive increasingly long nectar spurs in columbine flowers. Nature 447:706-710

Wolfe LM, Krstolic JL (1999) Floral symmetry and its influence on variance in flower size. Am Nat 154:484-488

Wright SI, Kalisz S, Slotte T (2013) Evolutionary consequences of self-fertilization in plants. Proc R Soc B 280:20130133

Yang JY, Hodges SA (2010) Early inbreeding depression selects for high outcrossing rates in Aquilegia formosa and Aquilegia pubescens. Int J Plant Sci 171:860-871

Yang JIY, Counterman BA, Eckert CG, Hodges SA (2005) Crossspecies amplification of microsatellite loci in Aquilegia and Semiaquilegia (Ranunculaceae). Mol Ecol Notes 5:317-320

Zuur AF, Ieno EN, Walker NJ, Saveliev AA, Smith GM (2009) Mixed effects models and extensions in ecology with R. Springer, New York, NY 